\title{
Telugu based Emotion Recognition System using Hybrid Features
}

\author{
J. Naga Padmaja \\ JNTUH \\ HYDERABAD
}

\author{
R. Rajeswara Rao \\ JNTUK- UCEV \\ VIZIANAGARAM
}

\begin{abstract}
Emotion recognition from speech is experiencing different research applications. It is becoming one of the tool for analysis of health condition of the speaker. In this work, the emotions such as anger, fear, happy, neutral are considered for speech emotion algorithm design. A database built by IITKGP is used for emotion recognition. For any recognition, feature extraction and pattern classification are the important tasks. In this work the features considered are Mel Frequency Cepstral Coefficients (MFCC), Pitch chroma, prosodic are used. Hidden Markov Models (HMMs ) are used to for modeling and identify the emotions. In this research work, the database considered for emotion recognition is taken in different combinations such as male training- female testing, male training-male testing, female training- female testing, female training-male testing. All these combinations are trained and tested with i-vector with GMM, linear Hidden Markov Models (HMMs) and Ergodic Hidden Markov Models(EHMMs) In almost all the cases, Ergodic Hidden Markov Models (EHMMs) method has shown significant improvement in recognition accuracy than i-vector with GMM and Linear Hidden Markov Models(HMMs)
\end{abstract}

\section{Keywords}

Emotion Specific, I-Vector, Gaussian Mixture Models, Prosody Features, Spectral Features, HMM, EHMM

\section{INTRODUCTION}

Distinctive data, for example, emotion, speaker, gender, dialect and so forth can be seen from speech signal. Increment in the human PC collaboration requests more research in speech handling, for example, speaker ID, emotion ID, Speech recognition and so on. The execution of the a speech recognition or a speaker identification framework [1,2] relies upon the extraction of appropriate features from the speech signals The mental state of an individual is estimated by his emotion. The flow of speech is affected by its emotion [1]. In speech recognition emotion recognition is crucial.

The basic challenge for present research in the speech technology is analysis and modeling individual variations of speech emotions.[2]. Factors like dialect, emotions and accent of the speech characterizes speakers. Prosody of the language plays prominent role in speaker recognition task [3].

In this present work, emotion recognition is performed for various blends of training and testing utterances. The emotions like happy, Fear, Anger and neutral are considered in this work.

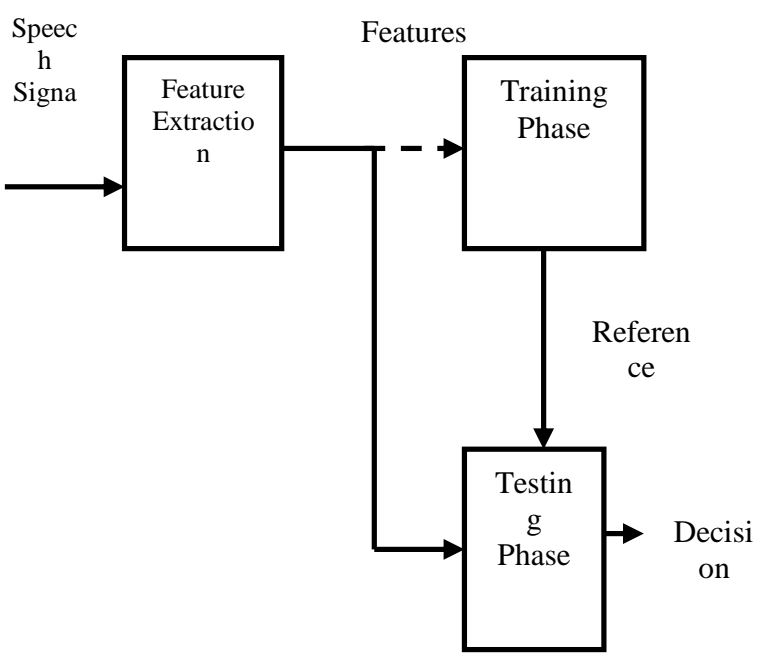

Fig. 1: Block diagram representing Emotion Recognition

\section{RELATED WORK}

Kasiprasad mannepalli et al have worked on emotion recognition system [5] that utilized MFCC features, pitch chroma, spectral flux and tonal power ratio. The classification methodology is improved by using fractional calculus along with Deep Belief networks. The performance of the system is considerably.

Laura Caponetti,Cosimo Alessandro Buscicchio and Giovanna Castellano [6] have taken a shot at emotion recogntion framework configuration in light of MFCC and Lyon cochlear models. The analyses were performed on SUSAS corpus. From perceptions made by them, the combinations for the Long Short Term Memory (LSTM) classifier with the Lyon cochlear portrayal gave better recognition performance when contrasted and consolidating a similar classifier with the customary MFCC portrayal.

Yazid Attabi and Pierre Dumouchel [7] have identified that, for the highly unbalanced classes of speech data, the back-end system increases the recognition accuracy obtained by using Gaussian Mixture Models with the application of proper sampling. The experiments demonstrated that anchor models such as Euclidean distance improved the performance of GMMs by 6.2 percent.

L. Zao [8] proposed the $\mathrm{pH}$ time-frequency vocal source feature for multi-style emotion classification in which binary acoustic mask also considered to increase the accuracy of emotion classification. Two different databases were used to test this methodology, the Berlin EMO-DB and SUSAS. The MFCC and the $\mathrm{pH}$ feature have shown improvement in accuracy with acoustic mask

In the design of emotion recognition system [9] different features Energy, different Formants, and Zero Crossing Rate (ZCR) have been used for the samples of different range (50 
or 100) for classification in the work "Realization of emotions in speech using prosodic and articulation features". The use of energy feature alone gave a recognition rate of $58 \%$ only. By adding formants and zero crossing rate, the recognition accuracy was increased to $78 \%$.

Mayank Bhargava and Tim Polzehl [10] have proposed their work on emotion recognition using rhythm and temporal features Apart from the features like MFCC's, pitch and energy/ intensity. The recognition accuracy of $80.60 \%$ on Berlin emotion database with 7 different emotions with speaker dependent framework was obtained.

Yasmine Maximos and David Suendermann- Oeft [11] have explored the recognition of emotions in speech via class of the FAU Aibo Corpus for the 2-class task. The database includes German speech recordings of fifty one children. They have examined special spectral and prosodic feature combination. They have taken into consideration 110 features and a feature size reduction technique was used. The parameter optimized sequential minimal optimization set of rules with Unweight averages recogntion (UAR) gave a recognition accuracy of $69.39 \%$. The Successive minimum Optimization (SMO) was said as better optimizer. The original overall performance of UAR turned into $63.8 \%$ and after adding, attributes selection and parameter optimization a growth in recognition accuracy by means of $5.6 \%$ is observed.

Ankur sapra, nikhil panwar and sohan panwar [12] have mentioned specific strategies for detection of human feelings is discoursed based totally at the acoustic features like pitch, energy and so on. The proposed gadget is the usage of the traditional MFCC technique after which the usage of nearest neighbor for the classification. The MFCC capabilities were extracted from the speech samples after the speeches were separated for male and woman. The recognition accuracy obtained by them in this proposed technique become $90 \%$.

Vaishali M. Chavan and V.V. Gohokar [13] used Support Vector Machine (SVM), to categorize five emotional states i.e anger, happiness, disappointment, surprise and a neutral emotion of human speech. The functions taken into consideration in their work have been Mel Frequency Cepstrum Coefficients. The database used was the Danish Emotion Speech (DES) Database. The accuracy acquired through using SVM classifier have been $68 \%$ for Linear regression version, $60 \%$ for polynomial regression, $55.40 \%$ for RBF regression and $60 \%$ with sigmoid regression. Overall time taken through SVM changed into 15 seconds for testing records set and for the take a look at pattern it turned into 43 seconds to test 88 values.

From the literature review, it is unmistakable that, most of the emotion studies using speech are conceded using prosodic features [14]. Pitch, duration and energy and their derivatives related Prosodic features are treated as high correlates of emotions [15].

Fundamental Frequency (F0), raise and falls of F0 contours measures and characterizes the emotions [16]. n [17][18] completely different emotions and there importance within the context of delivery options is mentioned. In varied dynamic nature of delivery contours [19] clearly provides varied expressions associated with emotions. Perception of high arousal emotions and low arousal emotions is achieved victimization MFCC, ZCR and Pitch [20].

Diana Torres-Boza et al [21] proposed method Hierarchical Sparse Coding (HSC) and Global Descriptor Extraction Layer (GDEL) is a combinational technique of Transfer Principle
Component Analysis (TPCA) and Sparse Coding Learning for emotion recognition. From the labeled and unlabeled data sparse coding stage set to learn robust feature representation. The experimental results of the proposed method gives $73 \%$ of accuracy in emotion recognition and by using the combinational method valence and arousal features emotion prediction is enhanced to $78 \%$ of accuracy.

Jainath Yadav, ,Md. Shah Fahad et al [22]. Due to rapid changes of pitch period in emotional speech performance degradation is more in the existing epoch estimation algorithm. In the proposed work zero time windowing method is utilized to get the spectral information of each sample point instantaneously. Compared to the neighboring sample points the amplitude of instant spectral of windowing technique is higher. The proposed method uses the sum of three prominent spectral peaks at each sampling instant of the Hilbert envelope of Numerator Group Delay (HNGD) spectrum, for accurate detection of epochs in the emotional speech. The identification rate (IDR) of epoch extraction is significantly high in the proposed method. The average values of IDR, RMSE, and IDA of detected epochs using proposed method are $93.69 \%, 0.42 \mathrm{~ms}$, and $0.30 \mathrm{~ms}$, respectively for the emotional speech.

Zhen-Tao Liu, et al [23].Feature vectors are set by extracting the test samples of speech emotional data., to realize the classification experiment these set are respectively input into the ELM decision tree and SVM decision tree. The time of accomplish speech emotion recognition and the accuracy of the two groups of experiment, in which the recognition time is the time from the input of the selected feature vector set to the output of the result. When using the feature set without feature selection, the average recognition accuracy of ELM decision tree is up to $88.251 \%$, which is about $1.2 \%$ higher than SVM decision tree. And recognition time of ELM decision tree is about $1.2 \mathrm{~s}$ less than SVM decision.

ShaolingJing et al [24]. This research proposes a novel type of feature related to prominence, which, together with traditional acoustic features, are used to classify seven typical different emotional states. A Chinese Dual-mode Emotional Speech Database (CDESD), which contains additional prominence and paralinguistic annotation information. Then, a consistency assessment algorithm is presented to validate the reliability of the annotation information of this database. The results show that the annotation consistency on prominence reaches more than $60 \%$ on average. The proposed prominence features are validated on CDESD through speaker-dependent and speakerindependent experiments with four commonly used classifiers. Consistency increases with increasing tolerance. For a time difference tolerance of $5 \mathrm{~ms}$, annotators is higher than $60 \%$ on average. When the time difference tolerance is increased to $8 \mathrm{~ms}$ and $10 \mathrm{~ms}$, the consistency increases by $6.5 \%$ and $10.5 \%$, respectively. $70 \%$ the low performance achieved in categorical emotion recognition is probably the monetization of emotion as distinct and independent affective states.

M. Srikanth, et al [25]. In this model Mel frequency Cepstral Coefficient (MFCC) and Gaussian Mixture Model (GMM) is used to extract the emotion independently. The proposed model GMM-DBN system by testing on the Classical German database (EmodB). Bag of acoustic features $(\mathrm{BoF})$ is derived by identifying the minimum distance distribution for individual utterance regarding emotion and histogram plotting count gives the feature distribution that is near to each emotion model. The performance rate increase by $5 \%$ than the 
conventional MFCC-GMM system by empirical observation. Further testing of the proposed system over the recently developed simulated speech emotion database for Tamil language gives a comparable result shows $90 \%$ accuracy for the emotion recognition.

Dai et al. [26] proposed a novel approach for emotional speech recognition and to analyze the voice content posted in social media like Wechat. By dynamic and emotional variations in Position-arousal-dominance (PAD) 25 acoustics voice signals are extracted and least squares-support vector regression (LV-SVR) model is used for classification of speech emotion. From the experimental results the recognition range for different emotions varies and the final average rate of recognition achieved is around $83 \%$. Which has the high accuracy rate than previous models.

Cao et al. [27] in order to reduce the error in binary classification, SVM ranking model is established to synthesize information of emotion speech recognition. To apply muticlass prediction each utterer is considered as individual query and then gathering all predictions from rankers for particular emotion. The major advantage of Ranking SVM method is to obtain speaker specific data for training and testing steps in speaker - independent and each speaker can express mixed emotion to recognize the dominant emotion. The proposed method attains gain in terms of accuracy when compared with conventional SVM. Unweight average (UA) or Balance accuracy achieved is $44.4 \%$.

\section{METHODOLOGY OF THE PROPOSED APPROACH}

Basically in view of the investigations completed in going before stage, it's miles introduced that the spectral features and prosodic features have two exceptional discriminative qualities. Along these lines to find the novel emotion specific data, it's far proposed to combine both spectral and prosodic features. For spectral features MFCC, Pitch Chroma, Spectral Centroid, and Spectral Skewness are viewed as and for prosodic features Pitch, Formants, Zero Crossing Rate, Root Mean Square and Energy are considered. The philosophy of the proposed approach is appeared in Fig 2. The database considered in this paper is IITKGP database. The speeches were separated for both the training and testing sets in the emotions of anger, Fear, Happy and Neutral. Both spectral and prosodic features are extracted and these features are concatenated. I-vectors extracted from the means of Gaussian Mixture Model (GMM) and are applied for the recognition of emotions for different cases.

\subsection{Procedure For Feature Extraction}

The important task in emotion recognition system is the extraction of best suitable features which represents acoustic data.

\subsubsection{SilenceRemoval}

The speech signal consists of both silence and voiced regions. The silent regions are identified and removed to obtain the exact feature from emotion speech database

\subsubsection{Pre Processing}

The speech signal identified with every emotion is preprocessed before demonstrating. Here, the given emotion speech test is pre-emphasized, blocked, windowed and examined at 16000 samples/sec. a frame size of $25 \mathrm{~ms}$ is considered with a shift of $10 \mathrm{~ms}$. Amplitude check is ascertained for the given speech signal to see if features are to be extracted or not. The speech signal is pre-emphasized and the windowing is performed using hamming window.

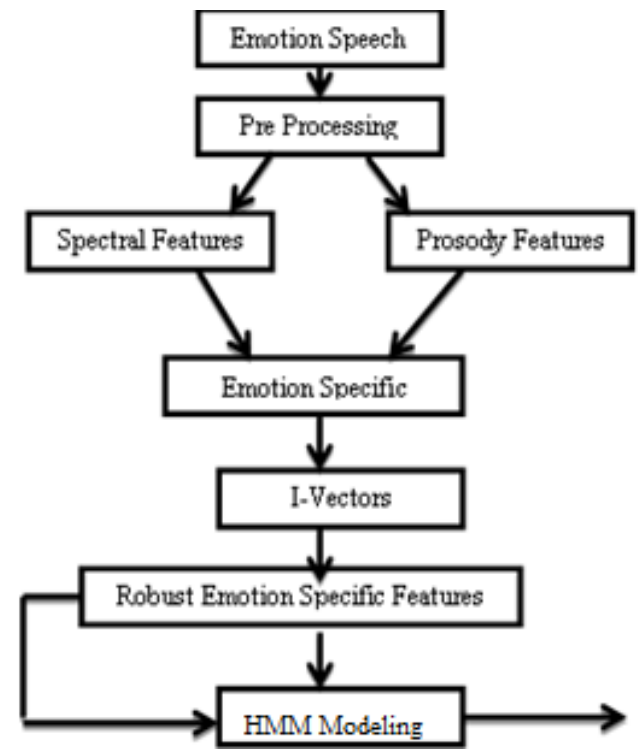

Fig. 2: Emotion Recognition System.

The pre-emphasize filter can be given by equation 1 .

$s^{\prime(t)}=s(t)+\alpha^{*} s(t-1)$

The typical $\alpha$ value is taken between 0 and 1 .

Generally hamming window is used for segmenting and selecting a part of speech signal. The Hamming window can be represented mathematically as follows in the equation 2 .

$w(l)=(1-a)-a \cos \left[\frac{2 \pi l}{N-1}\right]$

Here $\mathrm{a}=0.46164$ and where $\mathrm{N}$ is the length of window.

\subsubsection{Pitch Chroma}

The extraction of pitch chroma is the powerful tool to categorize the pitches significantly. On an equally tempered scale, the frequency of the Fourier transform is drawn with the 12 semi-tones pitch class C. Due to this, the transients are reduced and noise is minimized [21]. The pitch chroma is obtained as shown in the equation 3 .

$C(l)=\sum_{n^{\prime} ; c\left(n^{\prime}=l\right)} \sum_{f} h_{n^{\prime}}(f) X(f) ; l \in[0,1,2]$

Where, $X(f)$ is the Fourier transform of input speech signal. The filter $\mathrm{hn}(\mathrm{f})$ is defined by the following equation. separation between spectrum frequency and centre of the filter is represented by parameter $\mathrm{x}$

$$
h_{n^{\prime}}(f)=\frac{1}{2} \tanh (\pi(1-2 x))+\frac{1}{2}
$$

Where,

$$
x=R\left|n^{\prime}-n(f)\right|
$$

\subsubsection{Mel Frequency Cepstral Coefficients}

The human audio frequency bands can be extracted by Mel Frequency Cepstral Coefficient (MFCC) [22, 23]. The DFT is computed for the speech segments using the window function defined in earlier section and thus the power spectrum is found. The power spectrum is converted into Mel spectrum by 
triangular filter banks called as Mel filter banks. Further, the energy $G$ is calculated by multiplication of each filter bank with power spectrum and the logarithm is applied. MFCC features are obtained by application of the Discrete Cosine Transform (DCT) on the filter bank energies $G(p)$ for the $p \log$ filter bank energies is calculated as shown in equation 5 .

$$
\begin{aligned}
& M(p)=\frac{1}{2}\left(G_{0}+(-1)^{k} G_{p-1}+\sum_{q=1}^{M-2} G_{q} \cos \left[\frac{\pi}{m-1} q p\right]\right. \\
& \qquad m=0, \mathrm{~K}, M-1 \\
& \text { Where, } M(m) \text { is the desired } p_{\text {cepstral Coefficient. }}
\end{aligned}
$$

\subsubsection{PROSODIC FEATURES}

Prosodic features are supra segmental in nature which can happens in some top level of an speech utterance. These prosodic features require not identify with linguistic units, for example, phrases and clauses. Prosodic units are set apart by phonetic prompts which incorporate parts of prosody like Pitch, and Accents, that must be broke down in setting, or in contrast with different parts of a sentence. For instance, Pitch can change over the span of sentence, falling sounds. Prosody helps in settling sentence vagueness. The pitch related prosody is gotten via autocorrelation technique.

\subsubsection{FORMANTS}

The spectrum peak of the speech is called as the formant. The frequency components of human speech formants with F1, F2, $\mathrm{F} 3$. The arrangement of formants is from F1 to F3 in an increasing order. The formants generally distinguish the vowels. The LPC model is used to find the formants of a given speech signal in this research work. Linear Prediction Coding (LPC) method is used in estimation of formant estimation in light of the fact that the determination can be set by windowing the speech signal and obtaining the LPC coefficients

The mathematical expression for LPC can be shown in the zdomain with the equation 3 .

$$
H(z)=\frac{1}{1-\sum_{k=1}^{p} a_{k z^{-K}}}
$$

The poles of the vocal tract can be given by the roots of linear predication coding and the formants are associated with respective vocal tract poles.

\subsection{Hidden Markov Model I-vector}

Markov models are stochastic understandings of time arrangement. The essential Markov demonstrate is the Markov chain, which is spoken to with a chart made by a set out of $\mathrm{N}$ expresses; the diagram portrays the way that the likelihood of the following occasion relies upon the past occasion. The present state is transiently connected to $\mathrm{k}$ states in the past by means of an arrangement of $\mathrm{Nk}$ change probabilities. Give us a chance to mean the nonexclusive condition of the framework with St, St $\in\{1,2, \ldots, N\}$ and by $\mathrm{a}(\mathrm{St} \mid \mathrm{St}-1, \mathrm{St}-2, \ldots, \mathrm{St}-\mathrm{k})$ the likelihood that the framework is presently in state St given the already succession of states $\mathrm{St}-1, \mathrm{St}-2, \ldots . \mathrm{St}-\mathrm{k}$; an () is known as the progress likelihood for a model of request $\mathrm{k}$. In homogeneous Markov chains, the change likelihood rely upon the past state just; in such case the progress probabilities can be spoken to by a change lattice. In the event that the Markov chain is completely associated, or ergodic, each condition of the model can be come to from each other state in a solitary virtual time step. As respects the perceptible abilities of such models, we can state that the self circles depict a territory in the process. Different kinds of HMMs could better depict the factual properties of the watched process.

\subsubsection{Left-Right HMM}

Transition Matrix characterizes the behavior of HMM. As shown in Fig. 3 transitions are allowed to states whose index is less than the current index. Further the initial state probabilities have the following properties. For transition tion matrix $A=\left\{a_{i j}\right\}$. The property for a left-right model is $a_{i j}=0, \forall j<i$ indices is less than the current state which is shown in the Fig. 5.1. Further the initial state probabilities exhibits the following property

$\pi_{i}= \begin{cases}o & i \neq 1 \\ 1 & i=1\end{cases}$

$$
A=\left\{a_{i j}\right\}
$$

$$
=\left[\begin{array}{ccc}
a_{11} & a_{12} & a_{13} \\
0 & a_{22} & a_{23} \\
0 & 0 & a_{33}
\end{array}\right]_{3 \times 3}
$$

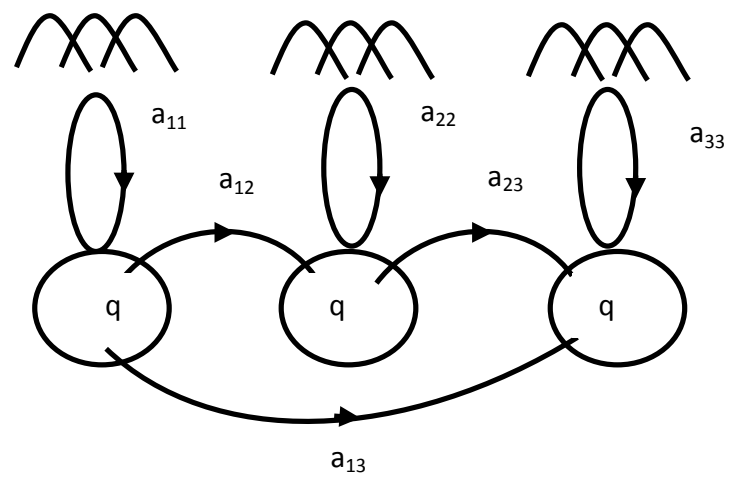

Fig. 3: A three state continuous left-right HMM

\subsubsection{Continuous Ergodic HMM: Its suitability for Emotion Recognition}

The ergodic model structure is defined by its transition matrix $A=\left\{a_{i j}\right\} \forall i, j$ an ergodic model is fully connected HMM. The ergodic model follows the property of complete graph. Here in this model every state can be reached from every other state of the model. The property of an ergodic HMM is given by $0<$ aij $<1$. The state transition matrix of three state ergodic model is given by 


$$
A=\left\{a_{i j}\right\}=\left[\begin{array}{lll}
a_{11} & a_{12} & a_{13} \\
a_{21} & a_{22} & a_{23} \\
a_{31} & a_{32} & a_{33}
\end{array}\right]_{3 \times 3}
$$

The underlying patterns of temporal sequence of emotional sound units and also non temporal sequences are effectively captured by continuous ergodic HMM. Therefore in order to capture both these type type of patterns ergodic HMMs are used for emotion recognition.

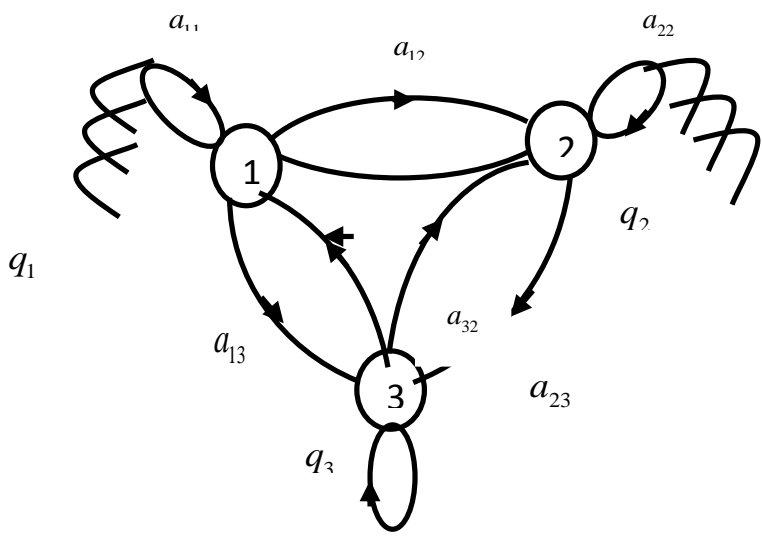

Fig. 4: A three state continuous Ergodic HMM

For instance, the left-to-right models have the property that, as virtual time expands, the state file additionally builds; they can along these lines display arrangements whose properties change after some time in a progressive way. As a rule, a homogeneous Markov chain has the accompanying properties: 1. restricted skyline: $\mathrm{P} \operatorname{rob}(\mathrm{St}+1 \mid \mathrm{St}, \mathrm{St}-1, \ldots, \mathrm{S} 1)=\mathrm{P}$ $\operatorname{rob}(\mathrm{St}+1 \mid \mathrm{St})$; 2. stationarity: $\mathrm{P} \operatorname{rob}(\mathrm{St}+1 \mid \mathrm{St})=\mathrm{P} \operatorname{rob}(\mathrm{S} 2 \mid \mathrm{S} 1)$. A Markov chain is in this way depicted by the change framework A whose components are ai, $\mathrm{j}=\mathrm{P} \operatorname{rob}(\mathrm{St}+1=\mathrm{j} \mid \mathrm{St}=$ I) and the underlying likelihood vector $\pi \mathrm{i}, \pi \mathrm{i}=\mathrm{P} \operatorname{rob}(\mathrm{S} 1=\mathrm{I})$, $\mathrm{PN} \mathrm{i}=1 \pi \mathrm{i}=1$. Be that as it may, by and large, Markov models are excessively straightforward, making it impossible to depict complex genuine frameworks and signs [8]. In Hidden Markov Models (HMMs), the yield for each state compares to a yield likelihood appropriation rather than a deterministic occasion. That is, if the perceptions are arrangements of discrete images browsed a limited letters in order, at that point for each state there is a relating discrete likelihood dissemination which portrays the stochastic process to be displayed. In HMMs, the state arrangement is hidden and must be seen through another arrangement of perceptible stochastic processes. In this way, the state arrangement must be recouped with a reasonable calculation, based on streamlining criteria. Note that the perception probabilities has been so far accepted discrete. Much of the time, in any case, the perceptions are ceaseless highlights vectors. It is conceivable to change over the ceaseless perceptions into discrete ones utilizing vector quantization, yet all in all some execution corruption because of the quantization process is watched. Subsequently, it is imperative, from an execution perspective, to utilize a general consistent formulation of the algorithms.

\section{FEATURE EXTRACTION OUTPUT}

The experimentations were done by combining both spectral and prosody feature

\subsection{EXPERIMENTAL SETUP}

The proposed Mixture models (GMMs) for modeling. 30 seconds of speech duration are considered for training and ten samples each of 3 seconds of speech duration is considered for testing. In this work the number of Gaussian Mixtures considered were 32 . The speech signal of anger emotion is shown in Fig. 5

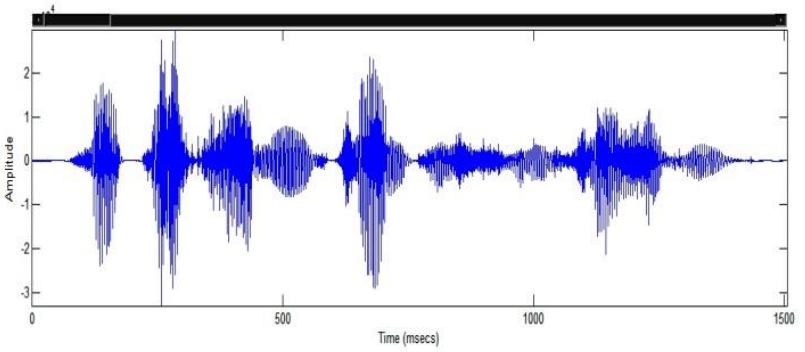

Fig 5.a: The speech signal of Anger emotion.

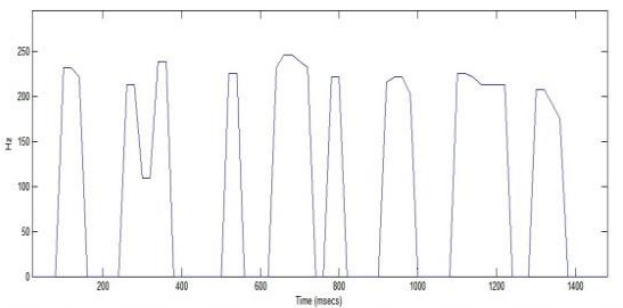

Fig 5.b: The pitch graph and related prosody for the signal in fig5.a

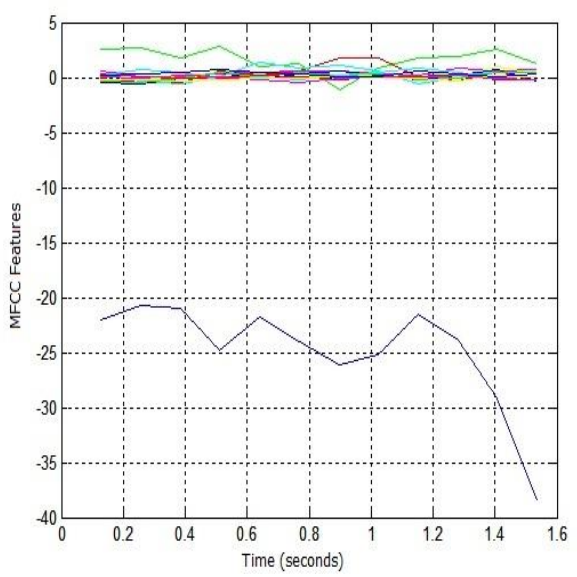

Fig 5.c: The MFCC feature graph for the signal in Fig 5.a 


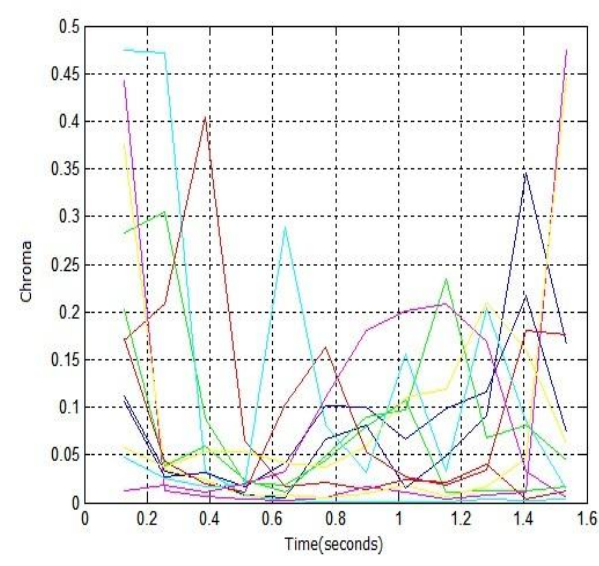

Fig 5.d: The pitch chroma for the signal in Fig 5.a

\section{DISCUSSION OF RESULTS}

In this research work, four emotions are considered for the analysis of the algorithm, those are anger, fear, happy and neutral. The research is carried out by taking the emotion speech sample in three different combinations of training and testing samples. The combinations are training with Male speech samples and testing with Male speeches termed as MM, The training with Male speech samples and testing with Female speeches termed as F-M. The training with Female speech samples and testing with Male speeches termed as MF. The training with Female speech samples and testing with Female speeches termed as F-F. In work i-vector are generated by joint feature analysis, by taking the means of Gaussian Mixtures.

The result is compared with PCA feature dimensionality reduction technique. When $\mathrm{M}-\mathrm{M}$ case is considered, the emotion recognition of anger has improved from $80 \%$ to $90 \%$, happy is improved from $70 \%$ to $80 \%$, neutral has improved from $80 \%$ to $90 \%$, whereas recognition accuracy of fear id in line with the PCA.

In the case $\mathrm{M}-\mathrm{M}$ is considered, the emotion recognition accuracy of anger has improved from $30 \%$ to $40 \%$, happy is improved from $30 \%$ to $40 \%$, and happy has improved from $40 \%$ to $50 \%$, whereas recognition accuracy of neutral is $50 \%$ which is in line with the PCA

Table 1: Comparison of Recognition accuracies for M-M case

\begin{tabular}{|c|c|c|c|c|}
\hline & Anger & Fear & Happy & Neutral \\
\hline $\begin{array}{c}\text { i-vector } \\
\text { Approach }\end{array}$ & 90 & 90 & 80 & 90 \\
\hline HMM & 93 & 90 & 80 & 93 \\
\hline E-HMM & 96 & 93 & 83 & 96 \\
\hline
\end{tabular}

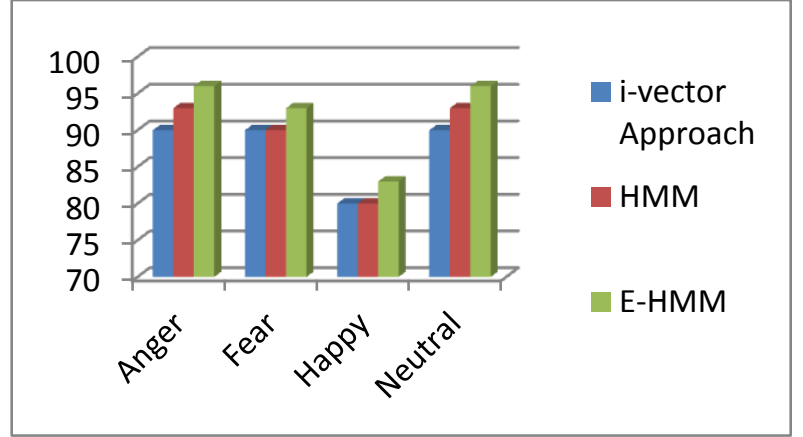

Fig. 6: Comparision of Recognition accuracies for M-M case

The graphical representation of the comparison of emotion recognition system for M-M case is depicted in Fig.6

In the case M-F, as shown in Table 2, the emotion recognition accuracy of anger has improved from $30 \%$ to $40 \%$, happy is improved from $30 \%$ to $40 \%$, and happy has improved from $40 \%$ to $50 \%$, whereas recognition accuracy of neutral is $50 \%$ which is in line with the PCA.

Table 2: Comaprision of Recognition accuracies for M-F case

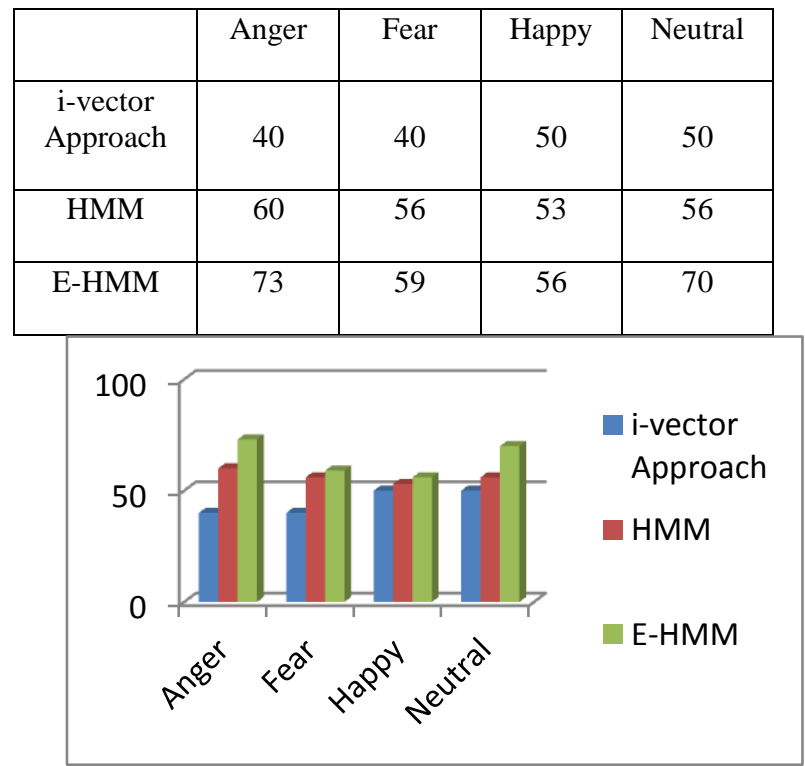

Fig. 7: Comparision of Recognition accuracies for M-F case

The graphical representation of the comparison of emotion recognition system for M-F case is depicted in Fig.7.

Table 3: Comparision of Recognition accuracies for F-M case

\begin{tabular}{|c|c|c|c|c|}
\hline & Anger & Fear & Happy & Neutral \\
\hline $\begin{array}{c}\text { i-vector } \\
\text { Approach }\end{array}$ & 60 & 50 & 50 & 60 \\
\hline HMM & 69 & 53 & 56 & 66 \\
\hline E-HMM & 75 & 59 & 62 & 72 \\
\hline
\end{tabular}




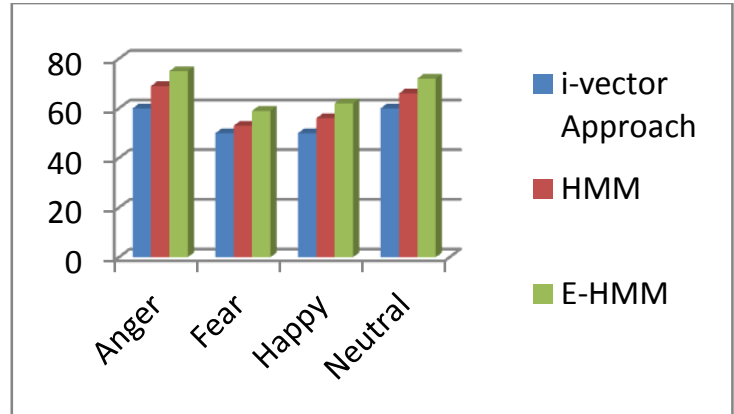

Fig. 8: Comparision of Recognition accuracies for F-M case

Table 4: Comparision of Recognition accuracies for F-F case

\begin{tabular}{|c|c|c|c|c|}
\hline & Anger & Fear & Happy & Neutral \\
\hline $\begin{array}{c}\text { i-vector } \\
\text { Approach }\end{array}$ & 100 & 90 & 100 & 80 \\
\hline HMM & 100 & 93 & 100 & 89 \\
\hline E-HMM & 100 & 96 & 100 & 96 \\
\hline
\end{tabular}

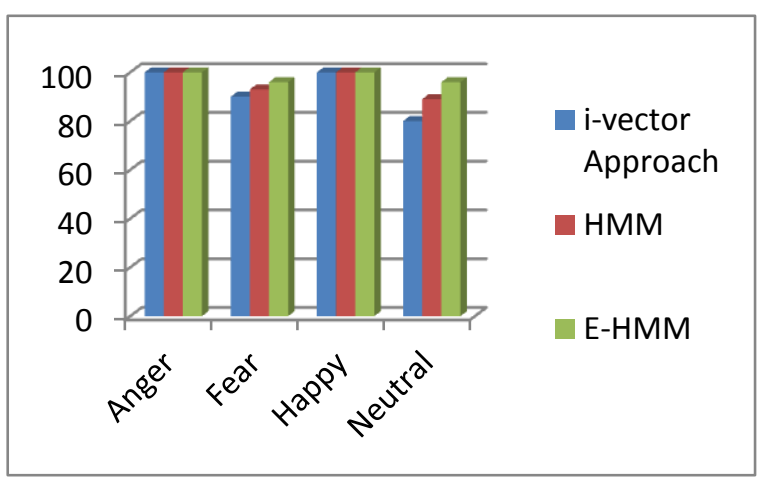

Fig. 9: Comparision of Recognition accuracies for F-F case

In the third case F-M, the emotion recognition accuracy of anger has improved from $40 \%$ to $60 \%$, fear is decreased from $60 \%$ to $50 \%$, and happy has improved from $40 \%$ to $50 \%$, whereas recognition accuracy of neutral is $60 \%$ which is in line with the PCA and this is shown in Table 3.

The comparison also shown graphically in the Fig. 8 for the F$\mathrm{M}$ case. Table 4 The emotion recognition accuracy of happy is improved from $90 \%$ to $100 \%$, neutral has improved from $60 \%$ to $80 \%$, whereas recognition accuracy of anger and fear have shown an accuracy of $100 \%$ and $90 \%$ respectively which are in line with the PCA.

\section{CONCLUSION}

1. Emotion specific features of the emotions Anger, Happy, Fear and Neutral are extracted from each emotion. The models are trained with the training sample duration of 30 seconds and tested with test samples of 3 seconds.

2. I-vectors are obtained from joint feature analysis of means.

3. There is a good improvement in the accuracies for the emotion recognition system for Gender dependent and Gender independent experiments by using Linear -HMM with respect to $\mathrm{i}$-vector.
4. From the experiments, it is observed that, Ergodic HMM has given better recognition performance than L-R HMM and i-vector.

\section{REFERENCES}

[1] Athanaselis et al.2005, "ASR for Emotional Speech: clarifying the issues and enhancing performance", Neural Netw, 18:437-444.

[2] Iker Luengo et al., 2008, "Text independent speaker identification in multilingual environments", lrec2008, pp:1814-1817.

[3] Ch.Srinivasa Kumar et al. "Design Of An Automatic Speaker Recognition System Using MFCC, Vector Quantization And LBG Algorithm", Vol.3, IJCSE,2011, pp:2942-2954.

[4] Prerna Puri, "Detailed analysis of Speaker Recognition System and use of MFCCs for recognition",Vol.3,2013, IOSR journal of Engineering, pp:32-36.

[5] Mannepalli, K., Sastry, P.N. \& Suman, M. Int J Speech Technol (2016) 19: 779. https://doi.org/10.1007/s10772016-9368-y

[6] Laura Caponetti,Cosimo Alessandro Buscicchio and Giovanna Castellano "Biologically inspired emotion recognition from Speech" Journal on Advances in Signal Processing, 2011.

[7] Yazid Attabi and Pierre Dumouchel, "Anchor Models for Emotion Recognition from Speech" IEEE Transaction on Affective Computing, Vol. 4, No. 3, pp: 280-290, 2013.

[8] L. Zão, "Time-Frequency Feature and AMS-GMM Mask for Acoustic Emotion Classification", IEEE Signal Processing Letters, Vol. 21, No. 5, pp: 620-624,May, 2014.

[9] S. Selva Nidhyananthan, R. Shantha Selva Kumari, L. Bala Manikandan \& P. Suresh, "Realiztion of emotions in speech using prosodic and articulation features" International Journal of Advanced Electrical and Electronics Engineering.Vol.2 Issue no.2, pp.83-86, 2013.

[10] MayankBhargava and Tim Polzehl "Improving Automatic Emotion Recognition from speech using Rhythm and Temporal feature" ICECIT published by Elsevier. Vol. 2 Issue no.2, pp.139-147, 2012.

[11] Yasmine Maximos and David SuendermannOeft,"Emotion recognition from children's speech" Speaker Emotion Challenge at Interspeech2013.

[12] Ankur Sapra, Nikhil Panwar and Sohan Panwar, "emotion recognition from speech",international journal of emerging technology and advanced engineering, volume 3, issue 2, pp. 341-345 February 2013.

[13] Vaishali M. Chavan and V.V. Gohokar "Speech Emotion Recognition by using SVM classifier" International Journal of Engineering and Advanced Technology (IJEAT), pp: 2249 - 8958, Volume-1, Issue-5, June 2012.

[14] Dellaert et al., " Recognizing Emotion in Speech", ICSLP, 1996.

[15] Lee CM, Narayanan, " Toward detecting emotion in spoken Dialogs”, IEEE Trans Speech Audio Process, 13 (2): 293-303 
[16] Murray, Arnott, "Implementation and Testing of a system by producing emotion by a rule in synthetic speech”, Speech Communication,16, 369-390.

[17] McGilloway et al. 2000, Rao et al. 2010," Approaching automatic recognition of emotion from voice", ISCA workshop on speech and emotion.

[18] Gish, H., Krasner, M., Russell, W., and Wolf, J., "Methods and experiments for text-independent speaker recognition over telephone channels," Proceedings of the IEEE International Conference on Acoustics, Speech, and Signal Processing (ICASSP), vol. 11, pp. 865-868, Apr. 1986.

[19] Reynolds, D. A., and Rose, R. C., "Robust TextIndependent Speaker Identification using Gaussian Mixture Models" IEEE-Transactions on Speech and Audio Processing, vol. 3, no. 1, pp. 72-83, Jan-1995.

[20] "Emotion Recognition using Prosody Features", Anne KR Kuchibotla, 2015.

[21] Diana Torres-Boza, Meshia Cedric Oveneke, et al. "Hierarchical Sparse Coding Framework for Speech Emotion Recognition" Speech Communication(2018), doi:10.1016/j.specom. 18.01.006.

[22] Jainath Yadav, ,Md. Shah Fahad et al. "Epoch detection from emotional speech signal using zero time windowing" Speech Communication 96 (2018) 142149.

[23] Zhen-Tao Liu, et al. "Speech emotion recognition based on feature selection and extreme learning machine decisiontree", Neurocomputing (2017),doi:10.1016/ j.neucom.2017.07.050
[24] ShaolingJing, XiaMao and LijiangChen. "Prominence features: Effective emotional features for speech emotion recognition". Digital SignalProcessing 017, doi.org /10.1016 /j.dsp. 2017.10.016.

[25] M. Srikanth, D. Pravena, and D. Govind. "Tamil Speech Emotion Recognition Using Deep Belief Network (DBN)" Advances in Signal Processing and Intelligent Recognition Systems, Advances in Intelligent Systems and Computing 678, 2018. DOI 10.1007/978-3-31967934-129

[26] W. Dai, D. Han, Y. Dai, and D. Xu, "Emotion Recognition and Affective Computing on Vocal Social Media,'Inf. Manag., Feb. 2015.

[27] H. Cao, R. Verma, and A. Nenkova, "Speaker-sensitive emotion recognition via ranking: Studies on acted and spontaneous speech," Comput. Speech Lang., vol. 28, no. 1,pp. 186-202, Jan. 2015.

[28] Peeters, G., "Chroma-based Estimation of Musical Key from Audio-Signal Analysis", In Proceedings of the 7th International Conference on Music Information Retrieval", Victoria (BC), Canada, 2006.

[29] Chin Kim On Paulraj M. Pandiyan Sazali Yaacob Azali Saudi, "Mel-Frequency Cepstral Coefficient Analysis in Speech Recognition", in proceedings of International Conference on Computing \& Informatics, pp. 1 - 5, 2006.

[30] Mannepalli, K., Sastry, P.N. \& Suman, M. Int J Speech Technol (2016) 19: 87. https://doi.org/10.1007/s10772015-9328-y. 